\title{
Coaxial Mono-Energetic Gamma Generator for Active Interrogation
}

\author{
B.A. Ludewigt ${ }^{1}$, A.J. Antolak ${ }^{2}$, E. Henestroza ${ }^{1}$, J.W. Kwan ${ }^{1}$, \\ M. Leitner ${ }^{1}$, K.-N. Leung ${ }^{1}$, W. Waldron ${ }^{1}$, S. Wilde $^{1}$ \\ ${ }^{1}$ Lawrence Berkeley National Laboratory, 1 Cyclotron Road, Berkeley, CA 94720 \\ ${ }^{2}$ Sandia National Laboratories, Livermore, CA 94550
}

\begin{abstract}
Compact mono-energetic photon sources are sought for active interrogation systems to detect shielded special nuclear materials in, for example, cargo containers, trucks and other vehicles. A prototype gamma interrogation source has been designed and built that utilizes the ${ }^{11} \mathrm{~B}(\mathrm{p}, \gamma){ }^{12} \mathrm{C}$ reaction to produce $12 \mathrm{MeV}$ gamma-rays which are near the peak of the photofission cross section. In particular, the ${ }^{11} \mathrm{~B}(\mathrm{p}, \gamma)^{12} \mathrm{C}$ resonance at $163 \mathrm{kV}$ allows the production of gammas at low proton acceleration voltages, thus keeping the design of a gamma generator comparatively small and simple. A coaxial design has been adopted with a toroidal-shaped plasma chamber surrounding a cylindrical gamma production target. The plasma discharge is driven by a $2 \mathrm{MHz}$ rf-power supply (capable up to $50 \mathrm{~kW}$ ) using a circular rfantenna. Permanent magnets embedded in the walls of the plasma chamber generate a multi-cusp field that confines the plasma and allows higher plasma densities and lower gas pressures. About 100 proton beamlets are extracted through a slotted plasma electrode towards the target at the center of the device that is at a negative $180 \mathrm{kV}$. The target consists of $\mathrm{LaB}_{6}$ tiles that are brazed to a water-cooled cylindrical structure. The generator is designed to operate at $500 \mathrm{~Hz}$ with 20 $\mu$ s long pulses, and a $1 \%$ duty factor by pulsing the ion source rf-power. A first-generation coaxial gamma source has been built for low duty factor experiments and testing.
\end{abstract}

Keywords: Photofission, Active Interrogation, Gamma Generator, Nuclear Reactions.

PACS: $52.38 . P h, 87.50 . G i, 29.17 .+w, 25.20 .-\mathrm{x}$

\section{INTRODUCTION}

Active interrogation with an intense source of highenergy photons or neutrons has a high probability of detecting shielded special nuclear material (SNM) with a minimal false alarm rate. Neutrons are effective for penetrating containers having strong photon-absorbing, high atomic number materials while high-energy photons have greater penetrability for cargo comprised of lighter elements, including hydrogenous materials, in which neutrons are readily absorbed. Mono-energetic photon sources have several advantages when compared to bremsstrahlung interrogation sources. With mono-energetic gammas, container and cargo are exposed to much lower radiation doses and, additionally, the photon intensity peaks at the desired energy for inducing photofission. This is in contrast to the exponentially decreasing bremsstrahlung energy spectrum with most photons well below the photofission threshold. Several promising light ion nuclear reactions have been identified that produce photons with energies above the photofission threshold. ${ }^{1}$ In particular, the
${ }^{11} \mathrm{~B}(\mathrm{p}, \gamma){ }^{12} \mathrm{C}$ reaction produces $12 \mathrm{MeV}$ gamma-rays which are close to the peak $(14 \mathrm{MeV})$ of the photofission cross section for the efficient stimulation of nuclear signatures. The ${ }^{11} \mathrm{~B}(\mathrm{p}, \gamma)^{12} \mathrm{C}$ resonance at $163 \mathrm{keV}$ allows the production of gammas at low proton acceleration voltages, thus keeping the design of a gamma generator comparatively small and simple. ${ }^{2}$

A new compact dual-particle generator has been designed that uses low-energy nuclear reactions to produce a high intensity source of neutrons and monoenergetic gammas for active interrogation applications. Pulsing the photon/neutron interrogation source makes it possible to take advantage of the full range of prompt \& delayed neutron and gamma fission signatures. ${ }^{3}$ High-yield systems exceeding $10^{10} \mathrm{n} / \mathrm{s}$ using D-D reactions have previously been designed and, therefore, the neutron part of the dual neutron/gamma source is essentially in hand. Based on the same concept, a novel gamma source utilizing the ${ }^{11} \mathrm{~B}(\mathrm{p}, \gamma){ }^{12} \mathrm{C}$ reaction has been developed and is described in the following sections. 


\section{COAXIAL GAMMA/NEUTRON SOURCE CONCEPT}

The coaxial gamma/neutron source concept is based on the idea of using low-energy nuclear reactions to produce the desired source radiation for interrogation. A compact generator can be realized by accelerating beam from a suitable plasma generator across a single gap onto a production target. For the gamma production, a proton beam impinges a ${ }^{11} \mathrm{~B}$ containing target while, for the neutron production, a deuteron beam and a beam-loaded deuterated titanium target are used. Lanthanum hexaboride $\left(\mathrm{LaB}_{6}\right)$ can be used as a boron-containing target. It is a rigid ceramic material with good thermal shock resistance and adequate electrical conductivity. Boron carbide $\left(\mathrm{B}_{4} \mathrm{C}\right)$ is another target material that can be tested in the future. However, this material is not a good conductor and, therefore, a thin film may need to be applied to prevent charging problem. The biggest challenge for the gamma source design utilizing the ${ }^{11} \mathrm{~B}(\mathrm{p}, \gamma){ }^{12} \mathrm{C}$ reaction is the need to operate with high proton current because of the small gamma production cross section $(0.16 \mathrm{mb})$ at its low-energy $163 \mathrm{kV}$ resonance. The gamma yield for the generator's nominal $180 \mathrm{kV}$ operating voltage using a $\mathrm{LaB}_{6}$ target has been roughly estimated as $4 \times 10^{8} \mathrm{\gamma} / \mathrm{C} .^{1,3}$ The design goals for the first test generator include a time-averaged beam current of $\sim 1 \mathrm{~A}$ at an acceleration voltage of $180 \mathrm{kV}$ and the capability of pulsed operation at $500 \mathrm{~Hz}$ and $20 \mu$ s pulse length for a duty factor of $1 \%$. The analogous design goals for neutron source operation include the same pulsing scheme with a $1 \%$ duty factor, but at a significant lower time-averaged current of $100 \mathrm{~mA}$ for the generation of $10^{11} \mathrm{n} / \mathrm{s}$ via the $\mathrm{D}(\mathrm{d}, \mathrm{n})^{3} \mathrm{He}$ reaction.

RF-driven ion sources have been shown to be capable of producing high extracted beam current with atomic ion species exceeding $90 \%{ }^{4}$ The high peak current needed for pulsed operation requires a large extraction area and a large volume ion source. For this reason, a coaxial design has been adopted with a toroidal-shaped plasma chamber with an inner diameter of $30 \mathrm{~cm}$ surrounding a cylindrical gamma production target shown schematically in figure 1. RFpower is coupled into the plasma through an internal antenna. The beam is extracted from the source (at ground potential) through a slotted grid with a large, total extraction area. The target is at high potential, which generates an electric field that extracts the ions from the plasma chamber and accelerates them across a single gap onto the target. An optional extraction electrode can be installed for gating the beamlets to achieve fast rise and fall times. The gamma production target consists of $\mathrm{LaB}_{6}$ sheets that are attached to a water-cooled backing. Magnetic field suppression is employed to prevent the back streaming of secondary target electrons. The target voltage is provided by a capacitor bank that is charged by a dc power supply.

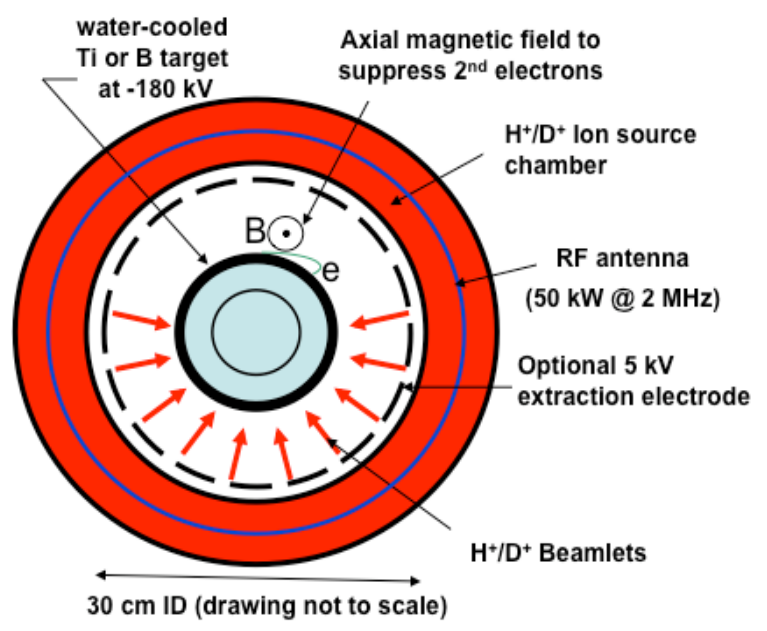

FIGURE 1. Coaxial gamma/neutron source concept.

\section{COAXIAL GAMMA GENERATOR DESIGN}

The coaxial gamma generator consists of a toroidal-shaped plasma chamber, shown in figure $2 \mathrm{a}$, surrounding a cylindrical gamma production target. The plasma discharge is driven by a $2 \mathrm{MHz}$ rf-power supply, capable of delivering up to $50 \mathrm{~kW}$, using an internal rf-antenna consisting of a conductor inside a water-cooled quartz tube. The antenna shape resembles a stretched winding that is bent around the toroidal chamber as seen in figure $2 \mathrm{~b}$. Permanent magnets embedded in all three walls of the plasma chamber (see figure 2a) generate a multi-cusp field that confines the plasma and allows higher plasma densities while operating at lower gas pressures.

The plasma chamber is at ground potential and a negative $180 \mathrm{kV}$ voltage is applied to the cylindrical target at the center of the generator for accelerating the ions toward the target. A $180 \mathrm{kV}, 267 \mathrm{nF}$ capacitor bank, built for pulsed operation, can supply a 100 A peak current for $20 \mu$ s with a $4.2 \%$ HV droop. Proton beamlets are extracted through 92 slits in the concentric plasma electrode that are $60 \mathrm{~mm}$ long and 5 $\mathrm{mm}$ wide resulting in a grid transparency of $\sim 50 \%$. The pressure in the acceleration gap needs to be in the low mTorr range to ensure HV holding. This is achieved by operating the ion source at low gas pressure $(<5 \mathrm{mTorr})$ and by pumping the acceleration 
gap with a turbo pump that is mounted on top of the generator chamber. A filament is provided for assisting plasma ignition and pulsed operation at low gas pressure.

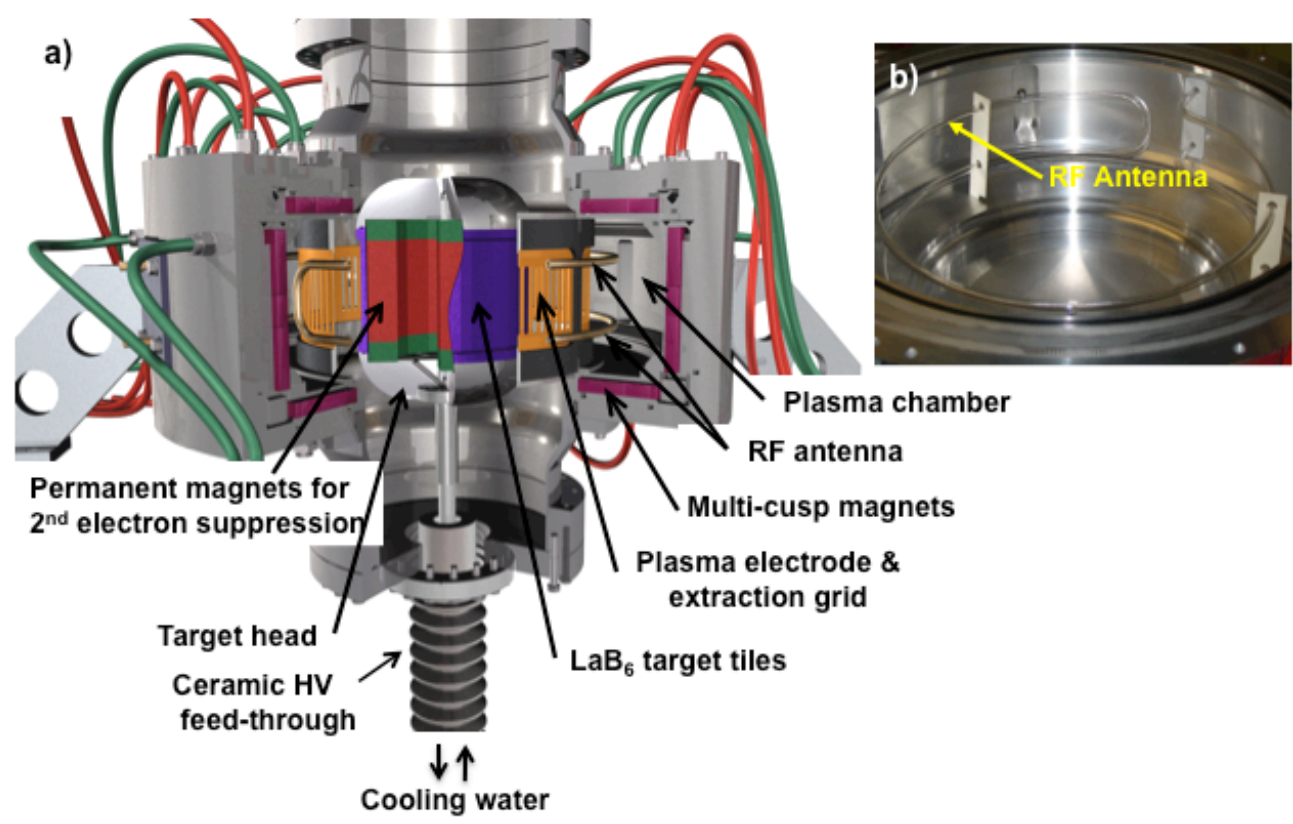

FIGURE 2. Gamma generator with toroidal-shaped plasma chamber and low-power gamma production target: a) schematic drawing, b) quartz RF antenna inside plasma chamber/neutron source concept.
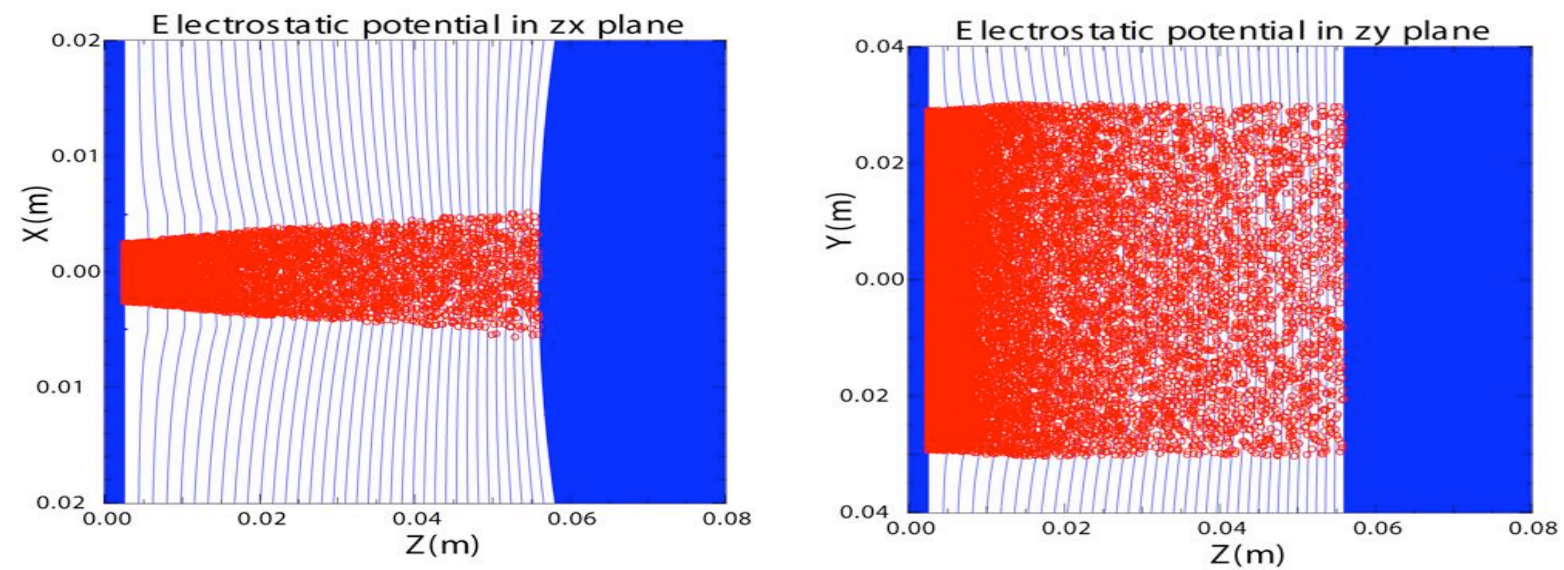

FIGURE 3. Computer simulation of the extraction and acceleration of a sheet beam (1 slit) from the ion source to the target. The left diagram shows the beam profile in the narrow slit direction, The beam profile in the wide direction is shown on the right.

Ion optics simulations were performed to calculate the ion trajectories of the extracted beamlets. ${ }^{5}$ The results, as shown in figure 3 , were used to determine the dimensions of the aperture slit, the acceleration gap distance, and the spot size on the target surface. The trajectories of secondary electrons emitted from the target surface in a suppressing magnetic field were also computed to optimize the field strength and the arrangement of the permanent magnets around the chamber.
As mentioned above the target consists of $\mathrm{LaB}_{6}$ tiles that are brazed to a water-cooled cylindrical structure. In particular, it was found that $\mathrm{LaB}_{6}$ could successfully be brazed onto a copper substrate. The target cylinder with a diameter of $20 \mathrm{~cm}$ is mounted on a stem in the center of the toroidal plasma chamber as shown in figure $2 \mathrm{a}$. The cooling water is brought into the target through a ceramic, vacuum-tight high voltage (HV) feedthrough. A low-power target was built for test operation at $1 \mathrm{~Hz}$. Finite element heat 
transfer calculations have been performed for the design of a high-power target consisting of $\mathrm{LaB}_{6}$ tiles brazed onto a thick copper backing. The calculation showed that the heat load from the full-power, pulsed ion beam (100 A peak current, 1\% duty factor) resulted in average and instantaneous temperatures of the $\mathrm{LaB}_{6}$ that were within acceptable limits.

\section{STATUS AND OUTLOOK}

The first-generation coaxial gamma source was designed and built for low duty factor experiments such that testing can be done with a low current 200 $\mathrm{kV}$ power supply at $1 \mathrm{~Hz}$ repetition rate. Figure 4 shows the device mounted in a teststand. The ion source was designed to deliver the desired high peak current for testing the basic operational functionality.

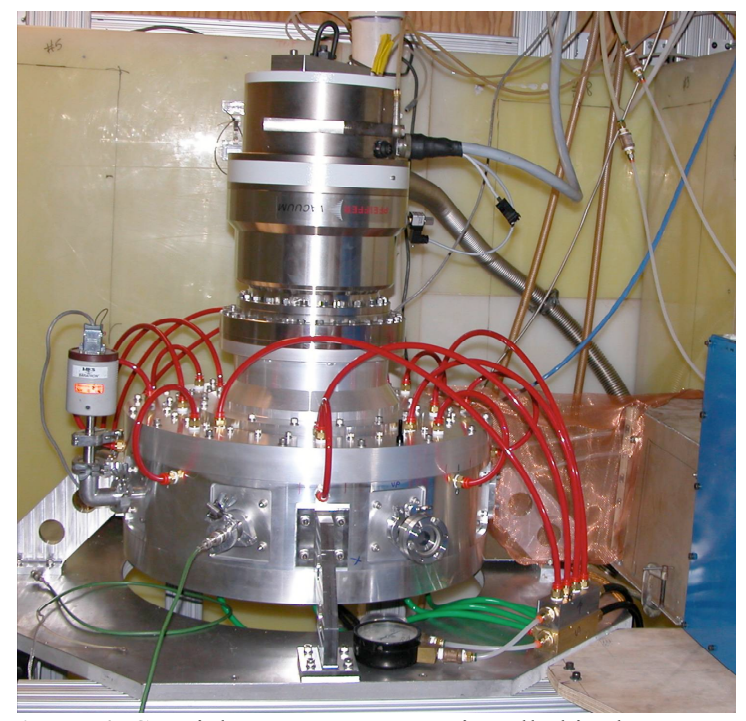

Figure 4. Coaxial gamma generator installed in the teststand. The lower, larger diameter chamber is the plasma chamber with the target mounted at its center. The turbo pump is seen mounted directly on top of it. The HV connection is from below.

In initial tests, the ion source could be ignited and operated in pulsed mode with $45 \mathrm{~kW}$ RF power at 8 mTorr hydrogen gas pressure. Further testing and optimization of low-pressure operation is needed. During high voltage conditioning of the apparatus 170 $\mathrm{kV}$ across the acceleration gap were reached with the ion source pressure at $8 \mathrm{mT}$, and with the target at positive polarity. High voltage holding was compromised when the target was at negative polarity, presumably due to the target's electron-suppressing toroidal magnetic field. It is planned to change the magnetic configuration into a multicusp field to eliminate the discharge problem. In conclusion, we have designed a gamma generator aimed at producing $\sim 10^{9} \mathrm{\gamma} / \mathrm{s}$ when using an optimized boron target. Higher gamma yields will likely require a device operating at accordingly higher beam currents and power levels.

\section{ACKNOWLEDGEMENT}

This work was supported by the US Department of Homeland Security, Domestic Nuclear Detection Office under contract number HSHQDC-07-00183 and by the Director, Office of Science of the US Department of Energy at Lawrence Berkeley National Laboratory under contract number DE-AC0205CH11231. Sandia National Laboratories is a multiprogram laboratory operated by Sandia Corporation, a Lockheed Martin Company, for the United States Department of Energy's National Nuclear Security Administration under contract DEAC04-94AL85000.

\section{REFERENCES}

1. A. J. Antolak, B. L. Doyle, K. N. Leung, D. H. Morse, P. Provencio, "Active Interrogation Using Low-Energy Nuclear Reactions", in Proceedings of SPIE: Penetrating Radiation Systems and Applications VII, v.5923, 2005, pp.1-9.

2. M. J. King, A. J. Antolak, K. N. Leung, D. H. Morse, T. N. Raber, "Performance of a Compact Gamma Tube Interrogation Source", to be published in AIP Conference Proceedings: CAARI2008, 2008.

3. D. H. Morse, A. J Antolak., B. L. Doyle. "Photofission in Uranium by Nuclear Reaction Gamma-Rays." Nucl. Instr. Meth. B261, 378-381 (2007).

4. J. H. Vainionpaa, K.N. Leung, R.A. Gough, and J. W. Kwan, "Ion source for neutral beam injection meant for plasma and magnetic field diagnostics", Review of Sci. Instrum. 79, $02 \mathrm{C} 102$ (2008).

5. D.P. Grote, A. Ihiedman, I. Haber, S. Yu, "ThreeDimensional Simulations of High Current Beams in Induction Accelerators with WARP3d", Fus. Eng. Des. 32, pp. 193 (1996). 